\title{
A RELATION BETWEEN $K$-THEORY AND COHOMOLOGY
}

BY

\section{ALAN THOMAS}

ABSTRACT. It is well known that for $X$ a CW-complex, $K(X)$ and $H^{\text {ev }}(X)$ are isomorphic modulo finite groups, although the "isomorphism" is not natural. The purpose of this paper is to improve this result for $X$ a finite $\mathrm{CW}$-complex.

1. Preliminaries. For the basic definitions and theory (1) of $\lambda$-rings, we refer to [2], [12]. The ring $Z$ of integers has a $\lambda$-ring structure with $\lambda^{m}: Z \rightarrow Z$ the function

$$
\lambda^{m}(n)=\left(\begin{array}{l}
n \\
m
\end{array}\right)=\frac{n(n-1) \cdots(n-m+1)}{m !}
$$

Definition. An augmented $\lambda$-ring is a $\lambda$-ring $R$ together with $\lambda$-ring homomorphisms $i: Z \rightarrow R$ and $\epsilon: R \rightarrow Z$ such that $\epsilon i=1$.

Since the Definition implies that $i: Z \rightarrow R$ is a monomorphism, we think of $Z \subset R$ as the multiples of the identity. Let $B$ denote the category of augmented $\lambda$-rings and $\lambda$-ring homomorphisms which commute with the augmentation. If $B \in$ $\mathrm{Ob} B$, we write $B_{n}^{\gamma}$ for the $n$th term of the $\gamma$-filtration on $B$, and $\Gamma(B)$ for the associated graded ring. We note that $\Gamma(B)_{0}=Z$. Let $\mathfrak{Q}$ be the category of commutative graded rings $A$ with $A_{0}=Z$. We define $\tilde{\Lambda}: \mathfrak{Q} \rightarrow \mathfrak{B}$ as follows: If $A \epsilon$ Ob $\mathbb{Q}$, then as a set $\tilde{\Lambda}(A)=\Pi_{n>0} A_{n}$. If $a \in \tilde{\Lambda}(A)$, we denote the component in $A_{n}$ by $a_{n}$ and for convenience of notation define $a_{0}=1 \in A_{0}$ and write $a$ as either of the formal expressions

$$
1+a_{1}+a_{2}+\cdots+a_{n}+\cdots \text { or } \sum_{i \geq 0} a_{i}
$$

If $a, b \in \tilde{\Lambda}(A)$, we define their sum, $a \oplus b$, componentwise:

$$
(a \oplus b)_{n}=\sum_{i+j=n} a_{i} b_{j}
$$

so that ' $\oplus$ ' is analogous with multiplication of formal power series. This operation makes $\tilde{\Lambda}(A)$ into an Abelian group, and we denote the inverse of $a$ by $\Theta a$. In particular if $a$ is an element with $a_{n}=0$ for $n \geq 2$, then

Received by the editors December 13, 1972 and, in revised form, July 9, 1973.

AMS (MOS) subject classications (1970). Primary 55B15; Secondary 55B40, 55F 40.

(1) We are indebted to Atiyah and Tall [2] for explicit proofs, in particular those based on 'formal al gebra'. 


$$
\ominus a=1-a_{1}+a_{1}^{2}-a_{1}^{3}+\cdots+\left(-a_{1}\right)^{n}+\cdots \cdot
$$

$A$ map $f: A \rightarrow B$ in $\mathscr{Q}$ induces a function $\tilde{\Lambda}(f): \widetilde{\Lambda}(A) \rightarrow \widetilde{\Lambda}(B)$ componentwise:

$$
\tilde{\Lambda}(f)(a))_{n}=f\left(a_{n}\right) \text {. }
$$

Since ' $\oplus$ ' is.defined in terms of the ring structure and $f$ preserves the ring structure, clearly $\tilde{\Lambda}(f)$ is a homorphism.

Define $\Lambda(A)=Z \oplus \tilde{\Lambda}(A)$ as a abelian group, and write $[m, a]$ for the element $m \oplus a$.

Proposition 1 (Grothendieck). (a) There is a unique multiplication on $\tilde{\Lambda}(A)$, denoted by $\otimes$, which is associative, commutative and distributive over addition such that

(i) for each integer $n$ there is a polynomial $P_{n}\left(X_{1}, \ldots, X_{n}, Y_{1}, \ldots, Y_{n}\right)$ with integer coefficients sucb that

$$
(a \otimes b)_{n}=P_{n}\left(a_{1}, \cdots, a_{n}, b_{1}, \cdots, b_{n}\right) ;
$$

(ii) if $a_{1}, b_{1} \in A_{1}$, then $\left(1+a_{1}\right) \otimes\left(1+b_{1}\right)=\left(1+a_{1}+b_{1}\right) \theta\left(1+a_{1}\right) \theta$

(b) If we give $\Lambda(A)$ the ring structure obtained from $A$ by adjoining a unit (i.e. define $[m, a] \otimes[n, b]=[m n, m b+n a+a \otimes b])$, then $\Lambda(A)$ admits a unique $\lambda \cdot$-ring structure such that

(i) for each pair of integers $m, n$, there is a polynomial $Q_{m, n}\left(X_{1}, \cdots, X_{n}\right)$ with integer coefficients such that

$$
\lambda^{m}[0, a]=[0, b] \text { where } b_{n}=Q_{m, n}\left(a_{1}, \cdots, a_{n}\right) ;
$$

(ii) $\lambda^{m}\left[0,1+a_{1}\right]=(-1)^{m-1}\left[0,1+a_{1}\right]$ if $a_{1} \in A_{1}$ and $m \geq 1$.

The existence and uniqueness depend on formal algebra.

We define a functor $D: \mathfrak{Q} \rightarrow \mathfrak{Q}$ as follows:

If $A \in \mathrm{Ob} \mathbb{Q}$ let $\times$ denote its multiplication. Define, $D(A)=A$ as a graded abelian group but with multiplication (denoted by $\cdot$ ) defined as follows: if $x_{m} \epsilon$ $A_{m}$ and $x_{n} \in A_{n}$ then

$$
x_{m} \cdot x_{n}=\frac{(m+n) !}{m ! n !} x_{m} \times x_{n}
$$

If $f: A \rightarrow B$ in $Q$ define $D(f)=f: D(A) \rightarrow D(B)$.

Let $N_{n}=N_{n}\left(\sigma_{1}, \ldots, \sigma_{r}\right)$ be the polynomial defined inductively for $n \geq r$ by $N_{1}\left(\sigma_{1}, \ldots, \sigma_{r}\right)=\sigma_{1}$ and the formula 


$$
N_{n}-\sigma_{1} N_{n-1}+\sigma_{2} N_{n-2}-\cdots+(-1)^{n} n \sigma_{n}=0 .
$$

Define $\sigma: \Lambda(A) \rightarrow D(A)$ by

$$
(\sigma[m, a])_{0}=m, \quad(\sigma[m, a])_{n}=N_{n}\left(a_{1}, \cdots, a_{n}\right) \text { for } n \geq 1,
$$

where $N_{n}$ is evaluated in $A$, not in $D(A)$.

Proposition 2. $\sigma: \Lambda(A) \rightarrow D(A)$ is a ring homomorphism.

Proof. That $\sigma$ is additive depends on certain identities satisfied by the polynomials $N_{n}$, and is omitted.

To show $\sigma$ preserves multiplication, by virtue of the universality of the definition of multiplication, it suffices to examine $\sigma(x \otimes y)$ where $x=[1,1+a]$ and $y=[1,1+b]$ with $a, b \in b_{1}$.

In this case $x \otimes y=[1,1+a+b]$ so that $(\sigma(x \otimes y))_{n}=(a+b)^{n}$. But

$$
(\sigma(x) \sigma(y))_{n}=\sum_{r+s=n} \frac{(r+s) !}{r ! s !} a^{r} b^{s}=(a+b)^{n} \text {. }
$$

So $\sigma(x \otimes y)=\sigma(x) \cdot \sigma(y)$.

2. The main theorem. Let $\mathbb{W}_{*}$ be the category of finite based connected $C W$ complexes and based maps. If $X \in \mathcal{W}_{*}$ then $H^{\mathrm{ev}}(X)=\bigoplus_{n \geq 0} H^{2 n}(X, \mathrm{Z})$ is a graded commutative ring and, since $H^{0}(X, \mathbf{Z})=\mathbf{Z}$, belongs to $\mathfrak{Q}^{n}$. Thus $H^{e v}, \mathfrak{W}_{*} \rightarrow \mathfrak{Q}$ is a functor, and we define

$$
\begin{aligned}
& \tilde{G}=\tilde{\Lambda}_{H}^{\mathrm{ev}}: \mathcal{W}_{*} \rightarrow \text { rings, } \\
& G=\Lambda H^{\mathrm{ev}}: \mathcal{W}_{*} \rightarrow \mathcal{B}, \\
& H=D H^{e v}: Q_{*} \rightarrow \mathbb{Q} \text {. }
\end{aligned}
$$

The internal multiplication $G(X) \otimes G(X) \rightarrow G(X)$ induces an extemal multiplication $G(X) \otimes G(Y) \rightarrow G(X \times Y)$ in the usual way. This in turn induces a multiplication $\tilde{G}(X) \otimes \tilde{G}(Y) \rightarrow \widetilde{G}(X \wedge Y)$.

If $E \rightarrow X$ is a complex vector bundle, let $c_{i}(E) \in H^{2 i}(X, Z)$ denote its $i$ th Chern class and define

$$
\begin{aligned}
& \tilde{c}(E)=1+c_{1}(E)+c_{2}(E)+\cdots \in \tilde{G}(X), \\
& c(E)=[\operatorname{rank} E, \tilde{c}(E)] \in G(X) .
\end{aligned}
$$

Lemma l. If $E, F$ are vector bundles over $X$ and $G$ over $Y$ then (1) $\tilde{c}(E \oplus F)=\tilde{c}(E) \oplus \tilde{c}(F)$ and $c(E \oplus F)=c(E) \oplus c(F)$. 
(2) $c(E \hat{\otimes} G)=c(E) \otimes c(G)$ where $E \hat{\otimes} G$ is the exterior tensor product bundle over $X \times Y$.

(3) $c\left(\lambda^{i} E\right)=\lambda^{i} c(E)$.

Proof. The formulae are the standard ones-see Hirzebruch [3].

Hence $\tilde{c}$ defines a ring homomorphism $\tilde{c}: \tilde{K}(X) \rightarrow \tilde{G}(X)$ and $c$ defines a $\lambda$ ring homomorphism $c: K(X) \rightarrow G(X)$. Let $s: K(X) \rightarrow H(X)$ be the composite $K(X)$ $\rightarrow^{c} G(X) \rightarrow^{\sigma} H(X)$.

The purpose of this section is to prove the following theorem. If $n$ is a positive integer let $l_{n}$ be the set of primes less than $n$.

Theorem 1. If $X$ is a finite CW.complex of dimension $\leq 2 n+1$, then

(i) $\tilde{c}: \tilde{K}(X) \rightarrow \tilde{G}(X)$ and $c: K(X) \rightarrow G(X)$ are isomorpbisms modulo $l_{n}$-torsion.

(ii) s: $K(X) \rightarrow H(X)$ is an isomorpbism modulo $l_{n+1}{ }^{-t o r s i o n . ~}$

The proof of Theorem 1 is an easy consequence of the following mod $\mathcal{C}$ version of a well-known theorem on half-exact functors.

Proposition 4. Let $\mathcal{C}$ be a Serre class of abelian groups, and let $\rho: t_{1} \rightarrow t_{2}$ be a map of balf-exact functors, where $t_{i}: W_{*} \rightarrow$ Abelian groups, sucb that $\rho:$ $t_{1}\left(S^{n}\right) \rightarrow t_{2}\left(S^{n}\right)$ is an isomorphism mod $C$ for $n \leq m$. Then $\rho: t_{1}(X) \rightarrow t_{2}(X)$ is an isomorphism mod $\mathcal{C}$ when $X$ is finite and $\operatorname{dim} X \leq m$.

Thus in order to prove the theorem, we examine the maps on spheres.

Lemma 2. (i) $\widetilde{K}\left(s^{2 n+1}\right) \rightarrow \widetilde{c} \widetilde{G}\left(S^{2 n+1}\right)$ is an isomorphism.

(ii) $\widetilde{K}\left(s^{2 n}\right) \rightarrow \widetilde{c} \tilde{G}\left(s^{2 n}\right)$ is a monomorphism with cokernel $\mathbf{Z}_{(n-1) !}$

(iii) $G\left(S^{2 n}\right) \rightarrow{ }^{\sigma} H\left(S^{2 n}\right)$ is a monomorphism with cokernel $\mathbf{Z}_{n}$.

Proof. (i) Trivial, since both groups are zero.

(ii) The map $K\left(S^{2 n}\right) \rightarrow G\left(S^{2 n}\right) \cong H^{2 n}\left(S^{2 n}\right)$ is given by the $n$th Chern class, and by theorems of Borel-Hirzebruch the $n$th Chern class of a complex vector bundle on $S^{2 n}$ is a multiple of $(n-1)$ ! times the generator, and every such multiple arises.

(iii) The map $G\left(S^{2 n}\right) \rightarrow^{\sigma} H\left(S^{2 n}\right) \cong H^{2 n}\left(S^{2 n}\right)$ is easily seen by calculation to be multiplication by $n$.

Let $\mathcal{C}_{n}$ be the class of abelian groups whose order is a product of primes in $l_{n}$. By Lemma 2

$$
\begin{aligned}
& \tilde{c}: \tilde{K}\left(S^{m}\right) \rightarrow \tilde{G}\left(S^{m}\right) \text { is an isomorphism mod } \mathcal{C}_{n} \text { for } m \leq 2 n+1, \\
& s: K\left(S^{m}\right) \rightarrow H\left(S^{m}\right) \text { is an isomorphism mod } \mathcal{C}_{n+1} \text { for } m \leq 2 n+1,
\end{aligned}
$$

whence the theorem follows. 
3. Finite $C W$-complexes of dimension $\leq 5$. In the case $n=2$, Theorem 1 says that if $\operatorname{dim} X \leq 5, c: K(X) \rightarrow G(X)$ is an isomorphism of $\lambda$-rings, i.e. $K(X)$ $\simeq \Lambda H(X)$ [in particular, if $\operatorname{dim} X \leq 4$, we see that $K^{1}(X) \simeq H^{1}(X) \oplus H^{3}(X)$ ], so that the graded ring structure of $H^{\mathrm{ev}}(X)$ determines the $\lambda$-ring structure of $K(X)$. In this section we show that in these low dimensions the converse is true, namely $H^{e v}(X) \cong \Gamma K(X)$. Since we already know that $K(X) \cong G(X)$ as $\lambda$-rings, it suffices to show that $\Gamma G(X) \cong H^{\mathrm{ev}}(X)$.

Let $a: \tilde{G}(X) \rightarrow H^{2}(X)$ be the projection $\alpha\left(1+a_{1}+a_{2}\right)=a_{1}$ and let $\beta$ : $H^{4}(X) \rightarrow \tilde{G}(X)$ be the inclusion $\beta\left(a_{2}\right)=1+\left(-a_{2}\right)$. The sequence $0 \rightarrow H^{4}(X) \rightarrow \beta$ $\tilde{G}(X) \rightarrow{ }^{a} H^{2}(X) \rightarrow 0$ is clearly exact.

Lemma 3. (i) $\operatorname{Im} \beta=G(X)_{2}^{\gamma}$. (ii) $G(X)_{n}^{\gamma}=0$ for $n>2$. (iii) The product in $\Gamma G(X)$ from $\left(G(X)_{1}^{\gamma} / G(X)_{2}^{\gamma}\right) \times G(X)_{1}^{\gamma} / G(X)_{2}^{\gamma} \rightarrow G(X)_{2}$ is (isomorphic to) the cup product $H^{2}(X) \times H^{2}(X) \rightarrow H^{4}(X)$.

Proof. By formal algebra it follows that

$$
\begin{gathered}
\gamma^{n}[0, a]=\left[0,1+(-1)^{n-1}(n-1) ! a_{n}+\cdots\right], \\
{\left[0,1+a_{n}+\text { higher terms }\right] \otimes\left[0,1+b_{n}+\text { hi gher terms }\right]} \\
=\left[0,1+c_{m+n}+\text { higher terms }\right] .
\end{gathered}
$$

From (1), (2), it follows that $G(X)_{n}^{\gamma}=0$ for $n>2$ and since $\gamma^{2}\left[0,1+a_{2}\right]=$ $\left[0,1-a_{2}\right]$, we see that $\operatorname{Im} \beta \subset G(X)_{2}^{\gamma}$. But $G(X)_{2}^{\gamma}$ in this case is generated by elements of the form $\gamma^{2}\left[0,1+a_{1}+a_{2}\right]$ or $\left[0,1+a_{1}\right]\left[0,1+b_{1}\right]$, i.e. by elements of the form $\left[0,1+a_{2}\right]$, whence $\operatorname{Im} \beta=G(X)_{2}^{\gamma}$. Finally a simple calculation shows

$$
\left[0,1+a_{1}+a_{2}\right] \otimes\left[0,1+b_{1}+b_{2}\right]=\left[0,1+\left(-a_{1} b_{1}\right)\right]
$$

which completes the proof of the lemma.

4. A real version. We would like to prove a theorem analogous to Theorem 1 for $\mathrm{KO}$-theory using the corresponding characteristic classes. Since Stiefel-Whitney classes do not carry enough information even on spheres, we try Pontryagin classes. However, there is a technical difficulty to overcome, namely that Pontryagin classes do not obey a Whitney-sum formula. However, if $E, F$ are real vector bundles over $X$ and if $p_{i}(E) \in H^{4 i}(X, Z)$ denotes the $i$ th Pontryagin class then $p_{n}(E \oplus F)-\Sigma_{i+j=n} p_{i}(E) p_{j}(F)$ is an element of order 2 in $H^{4 n}(X, Z)$. Let $H^{4}(X)=\bigoplus_{n \geq 0} H^{4 n}(X, Z)$. $H^{4^{*}}: W_{*} \rightarrow$ graded rings, so we can define

$$
(G O)^{\sim}(X)=\widetilde{\Lambda} H^{4 *}(X), \quad G O(X)=\Lambda H^{4^{*}}(X), \quad H^{4^{*}}(X)=D H^{4^{*}}(X) .
$$


(Alternatively, we can define $(G O)^{\sim}(X)$ as the subring of $\breve{G}(X)$ consisting of elements with zero components in odd degrees:

$$
(G O)^{\sim}(X)=\left\{a \in \tilde{G}(X): a_{n}=0 \text { if } n \text { is odd }\right\} .
$$

Let $q:(K O)^{\sim}(X) \rightarrow \widetilde{G}(X)$ be the composite

$$
q: K O(X) \stackrel{\text { complexification }}{\longrightarrow} K(X) \stackrel{c}{\longrightarrow} G(X) \stackrel{\psi^{2}}{\longrightarrow} G(X)
$$

where $\psi^{2}$ is the Adams operation.

Clearly $q$ is a ring homorphism.

Lemma 4. If $F \rightarrow X$ is a real vector bundle, then

$$
(q(E))_{2 n+1}=0, \quad(q(E))_{2 n}=(-4)^{n} p_{n}(E) .
$$

Proof. If $F$ is the complexification of $E$, with Chern classes $c_{1}, \cdots, c_{n}$, then $p_{i}(E)=(-1)^{i} c_{2 i}$. If $F$ were a sum of line bundles $F=L_{1} \oplus \cdots \oplus L_{n}$ with $c_{1}\left(L_{j}\right)=a_{j}$ then

$$
\begin{aligned}
\psi^{2} c(F) & =c \psi^{2}(F)=c\left(L_{1}^{2} \oplus \cdots \oplus L_{n}^{2}\right) \\
& =[1,1+2 \alpha] \oplus\left[1,1+2 a_{2}\right] \oplus \cdots \oplus\left[1,1+2 a_{n}\right] \\
& =\left[n, 1+2 c_{1}+2^{2} c_{2}+2^{3} c_{3}+\cdots\right]
\end{aligned}
$$

so by the splitting principle for complex vector bundles, we see that $(q(E))_{i}=$ $\left(\psi^{2} c(F)\right)_{n}=2^{n} c_{n}(F)$.

If $i$ is odd, then $2 c_{i}(F)=0$, so $(q(E))_{i}=0$. If $i=2 n$, then $(q(E))_{2 n}=$ $2^{2 n} c_{2 n}(E)=2^{2 n}(-1)^{n} p_{n}(E)$.

Thus the image of $q$ is contained in $G O(X)$. By naturality it induces a map $\tilde{q}:(K O)^{\sim}(X) \rightarrow(G O)^{\sim}(X)$.

Theorem 2. If $X$ is a finite $C W \cdot$ complex of dimension $\leq 4 n+3$, then

(i) $\tilde{q}:(K O)^{\sim}(X) \rightarrow(G O)^{\sim}(X)$ is an isomorphism modulo $\left(l_{2 n} \cup\{2\}\right)$-torsion.

(ii) $q: K O(X) \rightarrow G O(X)$ is an isomorphism modulo $\left(l_{2 n+1} \cup\{2\}\right)$-torsion.

(iii) $\sigma q: K O(X) \rightarrow D H^{4 *}(X)$ is an isomorphism modulo $\left(l_{2 n+1} \cup\{2\}\right)$-torsion.

Proof. As before it suffices to examine the maps on spheres. On $S^{t}$, where $t \equiv 0 \bmod 4,(K O)^{\sim}\left(S^{t}\right)$ is either $\mathrm{Z}_{2}$ or 0 , but $(G O)^{\sim}\left(S^{t}\right)$ and $H^{4 *}\left(S^{t}\right)$ are zero. The map from $(K O)^{\sim}\left(S^{4 n}\right) \rightarrow(G O)^{\sim}\left(S^{4 n}\right) \cong H^{4 n}\left(S^{4 n}, \mathrm{Z}\right)$ is $4^{n}$ times the $n$th Pontryagin class and again by theorems of Borel-Hirzebruch the $n$th Pontryagin class is a multiple of $(2 n-1) ! G C D(n+1,2)$, and moreover every such multiple arises. Thus this map from $Z$ to $Z$ is multiplication by some power of 2 times $(2 n-1)$ ! 
whose cokernel is thus a $\left(l_{2 n} \cup\{2\}\right)$-torsion group. The result now follows.

We briefly state two corollaries of Theorems 1 and 2 .

Corollary 1. (1) If $X$ is a finite $C W-$ complex of dimension $\leq 2 n+1$ and $H^{e v}(X)$ bas no $l_{n+1}$-torsion then

$$
K(X) \cong H^{\mathrm{ev}}(X) \text { as abelian groups. }
$$

(2) If $X$ is a finite $C W$-complex of dimension $\leq 4 n+3$ and $H^{4^{*}}(X)$ bas no $l_{2 n+1}$ torsion then

$$
K O(X) \cong H^{4^{*}}(X) \text { modulo } 2 \text { torsion, as abelian groups. }
$$

Proof. (1) $K(X)$ and $H^{e v}(X)$ have the same ranks and the same p-torsion for $p \equiv l_{n}$. From [6] by a simple spectral sequence argument we see that if $H^{\text {ev }}(X)$ has no $p$-torsion then $K(X)$ has no $p$-torsion.

(2) Proof similar.

Corollary 2. If $X$ is a finite $C W \cdot$ complex of $\operatorname{dim} \leq 2 n$ and $H^{2 k}(X)$ bas no $l_{k}$-torsion then $c: K(X) \rightarrow G(X)$ is an isomorphism on non- $l_{n}$-torsion and a monomorphism on $l_{n}$-torsion.

Proof. By Corollary 1 , it suffices to show that $\tilde{c}$ is a monomorphism. Suppose $\tilde{c}(x)=0$. Then since $\operatorname{dim} X \leq 2 n$, we can represent $x$ by $E-n$ for some $n$-dimensional complex vector bundle $E$. Then $\tilde{c}(E)=0$, so by a theorem of Peterson [7] $E$ is trivial whence $\tilde{c}$ is a monomorphism.

5. Bott periodicity and an exact sequence. By Brown's theorem, $\widetilde{K}$ and $\tilde{G}$ are representable functors. Let $\widetilde{K}()=[, E]$ and $\tilde{G}=[, B]$ where $E, B$ are $H$ spaces with multiplication $m$. Then $\tilde{c}: \widetilde{K} \rightarrow \tilde{G}$ defines a map $p: E \rightarrow B$ which we can assume without loss of generality to be a fibration, and which is an $H$-map, since $\tilde{c}$ is a homomorphism.

Let $i: F \rightarrow E$ be the fibre of $p: E \rightarrow B$. The composite $F \times F \rightarrow^{i \times i} E \times E$ $\rightarrow^{m} E \rightarrow^{p} B$ is homotopic to the composite $F \times F \rightarrow_{i \times i} E \times E \rightarrow_{p \times p} B \times B \rightarrow_{m} B$ which is the constant map. Let $H: * \simeq p m(i \times i)$.

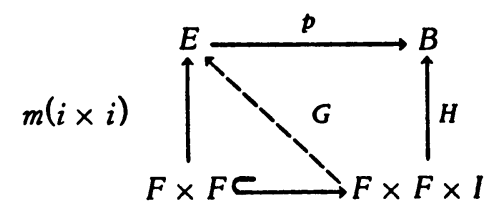

There exists a map $G: F \times F \times I \rightarrow E$ making the diagram commute. Let $m=G_{1}$ : $F \times F \rightarrow F$. Thus $m$ defines an $H$-space structure on $F$ such that $i: F \rightarrow E$ is an $H$-map.

Define $\tilde{U}=[, F]: \mathbb{U}_{*} \rightarrow$ Abelian groups. The Puppe sequence 


$$
\cdots \rightarrow \Omega^{n} F \stackrel{\Omega^{n} i}{\longrightarrow} \Omega^{n} E \stackrel{\Omega^{n} p}{\longrightarrow} \Omega^{n} B \rightarrow \ldots \rightarrow \Omega B \rightarrow F \stackrel{i}{\longrightarrow} E \stackrel{p}{\rightarrow} B
$$

induces a long exact sequence

$$
\begin{aligned}
\cdots \rightarrow\left[X, \Omega^{n} F\right] & \rightarrow\left[X, \Omega^{n} E\right] \rightarrow\left[X, \Omega^{n} B\right] \rightarrow \cdots \\
& \rightarrow[X, \Omega B] \rightarrow[X, F] \rightarrow[X, E] \rightarrow[X, B]
\end{aligned}
$$

which can be rewritten as

$$
\begin{aligned}
& \cdots \rightarrow \tilde{U}\left(\Sigma^{n} X\right) \rightarrow \tilde{K}\left(\Sigma^{n} X\right) \stackrel{\widetilde{c}\left(\Sigma^{n} X\right)}{\longrightarrow} \tilde{G}\left(\Sigma^{n} X\right) \rightarrow \cdots \\
& \rightarrow \widetilde{U}(X) \rightarrow \widetilde{K}(X) \stackrel{\widetilde{c}(X)}{\longrightarrow} \tilde{G}(X) .
\end{aligned}
$$

A simple calculation when $X=S^{k}$ gives the following lemma.

Lemma 5. (i) $\widetilde{U}\left(s^{2 n}\right)=0$.

(ii) $\tilde{U}\left(s^{2 n+1}\right)=Z_{n !}$.

(iii) If $X$ is a finite CW-complex of dimension $\leq 2 n+1$ with $\nu_{r}$ r.cells then $|\tilde{U}(X)|$ divides $\Pi_{r<n}(r !)^{\nu} 2 r+1$.

The purpose of this section is to obtain an exact sequence

(E)

$$
\tilde{U}\left(\Sigma^{2} X\right) \rightarrow K(X) \stackrel{s}{\rightarrow} H(X) \rightarrow \widetilde{U}(\Sigma X) \rightarrow K^{1}(X)
$$

$$
\stackrel{t}{\longrightarrow} H^{\text {odd }}(X) \rightarrow \tilde{U}(X) \rightarrow K(X) \stackrel{c}{\longrightarrow} G(X)
$$

where $t$ is defined as follows: If $E \rightarrow S X$ is an $n$-dimensional vector bundle, then it is determined by a map $X \rightarrow^{\prime} U(n)$. The cohomology of $U(n)$ is an exterior algebra on generators $x_{i} \in H^{2 i-1}(U(n))$ where $1 \leq i \leq n$. Define $t(E)=$ $\left(f^{*} x_{1}, f^{*} x_{2}, \cdots, f^{*} x_{n}\right)$.

First we observe that since cup products vanish on suspensions the natural bijection $\widetilde{H}^{\mathrm{ev}}(\Sigma X) \rightarrow \widetilde{G}(\Sigma X)$ is an isomorphism of abelian groups, so that $\widetilde{G}(\Sigma X)$ $\rightarrow H^{\text {odd }}(X)$, and $\widetilde{G}\left(\Sigma^{2} X\right) \cong \widetilde{H}^{\mathrm{ev}}(X)$, so we can insert these groups into the sequence (S). That the map

$$
t: K^{1}(X)=\tilde{K}(\Sigma X) \stackrel{\tilde{c}}{\rightarrow} \tilde{G}(\Sigma X) \cong H^{\text {odd }}(X)
$$

is given by the above construction we leave to the reader. Essentially it remains to prove the following lemma.

Lemma 6. The following diagram commutes: 


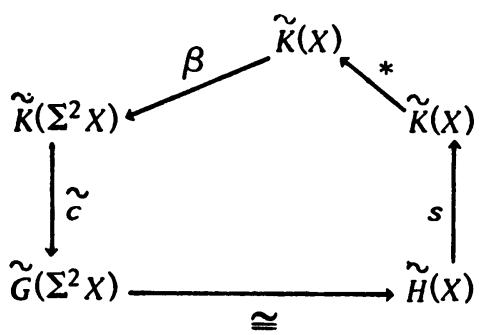

where $\beta$ is Bott periodicity and $*: \tilde{K}(X) \rightarrow \widetilde{K}(X)$ is conjugation.

Proof. Let $L \rightarrow X$ be a line bundle with $c_{1}(L)=l \in H^{2}(X)$ and let $H \rightarrow S^{2}$ be the Hopf bundle with $c_{1}(H)=b$. From the commutative diagram

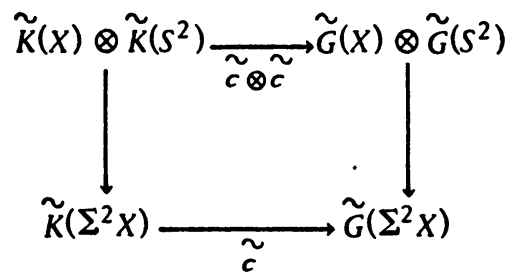

we see that

$$
\begin{aligned}
\tilde{c} \beta(L-1) & =\tilde{c}(L-1) \otimes \tilde{c}(H-1) \\
& =(1+l) \otimes(1+b) \\
& =(1+\hat{b}+\hat{l}) \ominus(1+\hat{b}) \ominus(1+\hat{l})
\end{aligned}
$$

where $\hat{b}_{,} \hat{l}$ are the images of $b, l$ in $H^{2}\left(s^{2} \times X\right)$. That is, $\tilde{c} \beta(L-1)=(1+\hat{b}+\hat{l})$ $\oplus(1-\hat{b}) \oplus\left(1-\hat{l}+\hat{l}^{2}-\hat{l}^{3}+\cdots\right)$ since $\hat{b}^{n}=0$ for $n>1$, i.e. $\tilde{c} \beta(L-1)=$ $(1+\hat{l}-\hat{b} \hat{l}) \oplus\left(1-\hat{l}+\hat{l}^{2}-\cdots\right)$. The component in dimension $n$, that is in $H^{2 n}\left(s^{2} \times X\right)$, is

$$
(-1)^{n-1} \hat{l}^{n-1} \hat{l}-(\widetilde{b} \hat{l})(-1)^{n-2} \hat{l}^{n-2}
$$

The term $(-1)^{n-1} \hat{l}^{n}$ lies in $H^{2 n}\left(S^{2} v X\right)$ and so contributes nothing in $H^{2 n}\left(\Sigma^{2} X\right)$ and the term $(-1)^{n-1} \hat{b} \hat{l}^{n-1}$ on desuspending maps to $(-1)^{n-1} l^{n-1} \in H^{2 n-2}(X)$, so that

$$
\tilde{c} \beta(L-1)=\left(-l, l^{2},-l^{3}, \ldots\right)=s(L-1)
$$

and so by the splitting principle and the universal definition of $s$, we see that $\tilde{c} \beta *=s$.

The exact sequence $(E)$ is now obtained from (S) by replacing $\widetilde{K}\left(\Sigma^{2} X\right) \rightarrow \widetilde{c}$ $\tilde{G}\left(\Sigma^{2} X\right)$ by $K(X) \rightarrow^{s} H^{e v}(X)$ which is isomorphic to $\tilde{K}\left(\Sigma^{2} X\right) \oplus Z \rightarrow \tilde{c} \oplus 1 \tilde{G}\left(\Sigma^{2} X\right)$ $\oplus Z$ and so preserves exactness. 


\section{REFERENCES}

1. M. F. Atiyah and F. Hirzebruch, Vector bundles and homogeneous spaces, Proc. Sympos. Pure Math., vol. 3, Amer. Math. Soc., Providence, R. I., 1961, pp. 7-38. MR 25 \#2617.

2. M. F. Atiyah and D. $O$. Tall, Group representations, $\lambda$-rings and the J-homomorphism, Topology 8 (1969), 253-297. MR 39 \#5702.

3. F. Hirzebruch, Neue topologische Methoden in der algebraischen Geometrie, Ergebnisse der Math. und ihrer Grenzgebiete, Heft 9, Springer-Verlag, Berlin, 1956; English transl., Die Grundlehren der math. Wissenschaften, Band 131, Springer-Verlag, New York, 1966. MR 18, 509; 34 \#2573.

4. A. Dold, Halbexakte homotopiefunktoren, Lecture Notes in Math., vol. 12, SpringerVerlag, Berlin and New York, 1966. MR $33 \# 6622$.

5. A. Borel and F. Hirzebruch, Characteristic classes and homogeneous spaces. I, Amer. J. Math. 80 (1958), 458-538. MR $21 \# 1586$.

6. L. Hodgkin, On the K-theory of Lie groups, Topology 6 (1967), 1-36. MR 35 \#4950.

7. F. Peterson, Some remarks on Chern classes, Ann. of Math. (2) 69 (1959), 414420. MR 21 \#1593.

8. E. H. Brown, Cohomology theories, Ann. of Math (2) 75 (1962), 467-484. MR 25 $\# 1551$.

9. J. P. Serre, Groupes d'homotopie et classes de groups abéliens, Ann. of Math. (2) 58 (1953), 258-294. MR 15, 548.

10. A. Grothendieck, Special $\lambda$-rings, (1957) (unpublished).

11. A. Thomas, Almost complex structures on complex projective spaces, Trans. Amer. Math. Soc. 193 (1974), 123-132.

12. D. Knutson, $\lambda$ rings and the representation theory of the symmetric group, Lecture Notes in Math., vol. 308, Springer-Verlag, Berlin and New York, 1973.

DEPARTMENT OF MATHEMATICS, UNIVERSITY COLLEGE OF SWANSEA, SINGLETON PARK, SWANSEA, WALES 